\title{
In Situ UV Fiber Optics for Dissolution Testing - What, Why, and Where We Are After 30 Years
}

\author{
Ishai $\mathrm{Nir}^{1, *}$ and Xujin Lu² \\ ${ }^{1}$ Distek, Inc. North Brunswick, NJ, USA \\ ${ }^{2}$ Bristol Meyers Squibb, New Brunswick, NJ, USA
}

\section{ABSTRACT}

e-mail: ishai.nir@distekinc.com

Ultraviolet (UV) fiber optic spectroscopy has been applied to dissolution testing for more than 30 years. Multiple types of instrumentation schemes have been designed and implemented over that time, all aimed to introduce UV light into the dissolution apparatus to perform in situ measurement of the percent dissolved of the active pharmaceutical ingredient (API). In situ UV fiber optic systems provide many advantages over conventional dissolution testing. It generates realtime dissolution profiles with more frequent data points, providing rich information useful not only for regulated quality control tests, but also for formulation development, troubleshooting, and analysis of novel formulations, such as nanoparticles that are hard to filter. In situ UV measurement without filtration is challenging due to the effects of particulates and scattering. Mathematical "filters" including baseline correction and derivative spectroscopy can overcome these challenges and remove the contribution of non-API components. Innovative applications such as multicomponent dissolution, small volume bio-predictive dissolution, and biphasic dissolution are also being advanced by using in situ UV testing. More benefits are expected as the in situ UV methodology for dissolution is more broadly accepted and implemented in pharmaceutical arena.

KEYWORDS: UV fiber optics, dissolution, in situ measurement, multicomponent analysis

\section{INTRODUCTION}

Itraviolet absorbance spectroscopy (UV) was one of the earliest ways to measure active pharmaceutical ingredient (API) percent dissolved values in dissolution testing, and it is still one of the most common methods. The traditional method involved removing an aliquot from the dissolution apparatus at time points of interest and bringing it for analysis to the UV spectrophotometer, either manually or using some form of automation. It has been more than 30 years since researchers have started to develop ways to perform the measurement in situ in the dissolution test apparatus using fiber optics and probes (1). Over that time, multiple schemes have been implemented, and all have been predicated on the idea that moving photons is much more convenient than moving liquid, making it possible for dissolution test users to perform measurements they always wanted to do but could not (2-6).

Over the years, conventional dissolution testing has been optimized to meet the requirements established by the various regulatory bodies. Yet it was well understood that the test itself had greater potential and could provide additional useful information beyond that required by the regulations for formulators, quality control (QC), and analytical services. In particular, if one could get a complete dissolution profile with adequate temporal resolution and number of data points, then one can use dissolution in ways beyond simple manufacturing validation and QC. Formulators could use this information to better understand ratelimiting steps of fast dissolving products. They can also consider implementing predictive dissolution testing, using the high data density of early times to accurately forecast the ultimate long-term dissolution results for development (and perhaps someday QC) of extended release formulations, reducing the duration and cost of such testing.

Similarly, QC can benefit from a technique that yields complete profiles and real-time results. Manufacturing and analytical services can use the information contained in a complete profile to better diagnose an Out of Specification (OOS) test. They can use the immediate results to alert operators of problems with a run sooner and avoid the time and cost of completing and analyzing samples from an obviously failed test. QC would also greatly benefit from dissolution automation that was less labor intensive and prone to error. 
These were the motivations for developing in situ UV testing. UV light could be introduced into the apparatus as often as desired and measured in real-time and without any disturbance of the hydrodynamics that would result from such frequent sampling. This enables immediate recording of percent dissolved values measured with extremely short intervals between time points and a very large number of readings. Eliminating sampling obviously removes sampling errors, such as incorrect sampling times, positions, filters, and handling. Additionally, it also prevents issues with carryover, cross contamination of filters and lines associated with automated liquid sampling, and the need for volume corrections due to media loss. Immediate in situ analysis also eliminates the possibility of partially filtered samples continuing to dissolve until the actual measurement can be made. And, because there is no filtering, there are fewer issues with analyzing hard-to-filter samples like nanoparticles (7). Other innovative applications being advanced by using in situ UV testing include USP apparatus 4, small volume bio-predictive dissolution, and biphasic dissolution (8-11).

\section{DESIGN OF IN-SITU UV SYSTEMS}

In situ UV testing is not a new idea. The first publication describing such a system was by researchers at AstraZeneca (then Astra Hässle) in Mölndal, Sweden in 1988 (1). The first system to be later commercialized was conceived by Walker at GlaxoSmithKline (then Burroughs Wellcome Co.) and developed into a working system in conjunction with Gemperline and Cho at East Carolina University in the early 1990s. The system was described by Walker et al in $1995(12,13)$. This was shortly followed by Bynum et al at Purdue Pharma, where a system was developed for in-house use (14). A company to commercialize the system was spun out of Purdue Pharma and eventually sold. These and other systems became commercially available in the late 1990s and early 2000s (15-17).

In situ UV systems share the majority of the elements of conventional spectrophotometers. There is a light source which supplies the UV and a detector (or detectors in some implementations) that measures the light transmitted through the sample volume. The difference from conventional UV spectrophotometry is that rather than a cuvette to hold the sample, there are fiber optics that transmit the light to and from the apparatus and in situ probes that define the sampling volume (path length). Multiple variations of the scheme have been utilized in designing these systems (18). These have mostly varied in the choice of detector and probe design. Some systems are simple single-channel designs only capable of measuring one vessel at a time. The majority, however, measure all the vessels at or near the same time. The simplest of these use a conventional scanning or diode-array-based spectrophotometer with a multiplexer to sequentially switch amongst the vessels (Fig. 1). This means that the dissolution is not measured truly simultaneously in each vessel, and the reproducibility of the multiplexer needs to be validated. To eliminate these problems, some systems opt to use multiple diode array detectors, one per vessel. This approach permits simultaneous measurement from all vessels but introduces the need to validate and crosscorrelate the multiple detectors, because it essentially the same as using multiple spectrophotometers to measure each vessel. To simultaneously measure all vessels with one detector, some systems use a multichannel charged couple device (CCD) detector and imaging spectrograph. In this design, the spectrum from each probe is created on a separate part of the two-dimensional detector, allowing one detector to act as multiple ones. Because the light from all vessels are collected in one optical system, care needs to be taken in the design of such systems to avoid crosstalk amongst the various vessels.

As is the case with detection schemes, there is also variation in design of the fiber optic probes (Fig. 2). Early systems used standard transmission dip probes designed for general purpose liquid measurement. Some of these probes had the nice feature of having interchangeable tips, which allow changing the pathlength, like selecting different cuvettes in a conventional UV system. Unfortunately, these also had several issues when applied to dissolution testing. In their normal use, there is no concern about hydrodynamics, so these probes tended to be thick (typically $\sim 6 \mathrm{~mm}$ ). Such large probes being resident during a dissolution test could affect the results, so implementation required either validation of the lack of such effects or the addition of a raising and lowering manifold to remove the probes from the media when not sampling (19-21). Another issue was that the vertical design meant that the probes were prone to trapping air bubbles and particulates. Also, the original designs, when used with pathlengths below $2 \mathrm{~mm}$ (which translates to a 1-mm opening because the light traverses the opening twice in these probes), would sometimes result in inadequate liquid flow through the probe, especially with viscous media or use of surfactants. Later generations of transmission probes addressed some of these concerns. Models became available with a diameter less than 3 $\mathrm{mm}$, much closer to that of cannulas conventionally used in dissolution testing. Also, the sampling cavity flow was improved by changing the design from having supports

Dissolution 

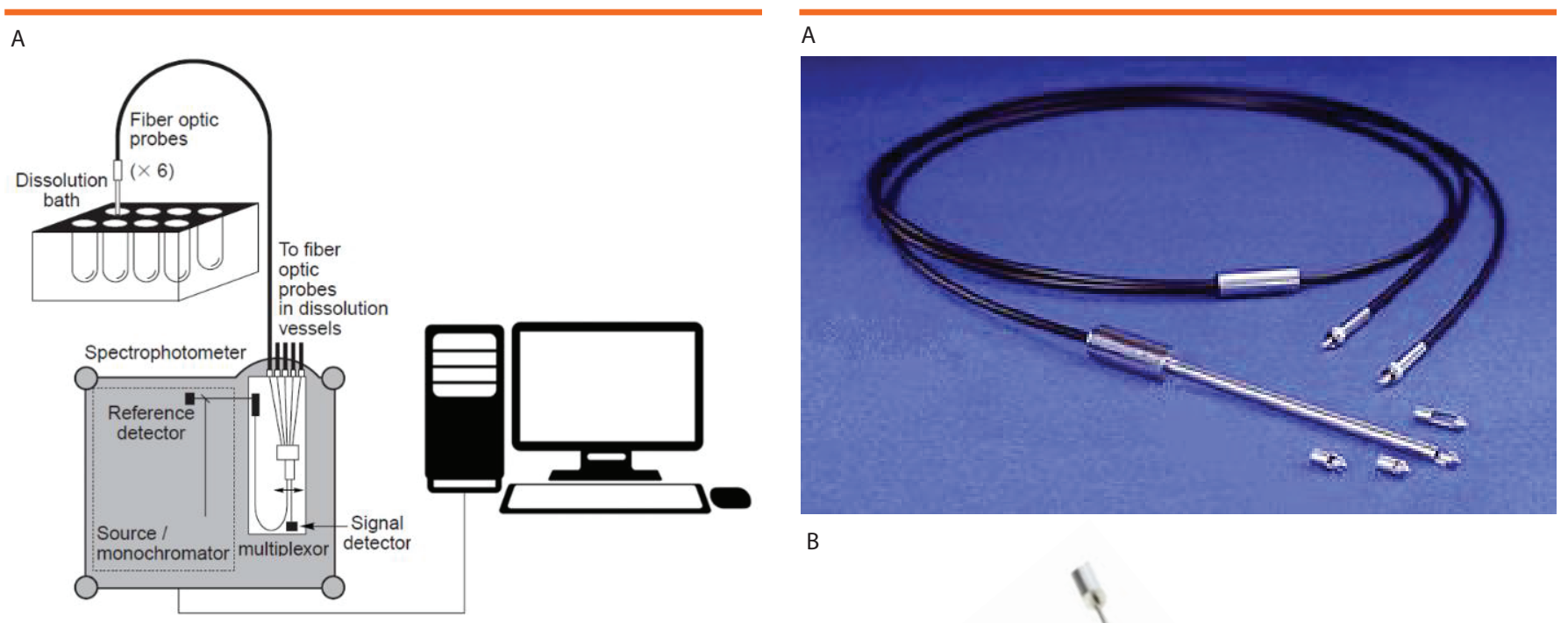

B

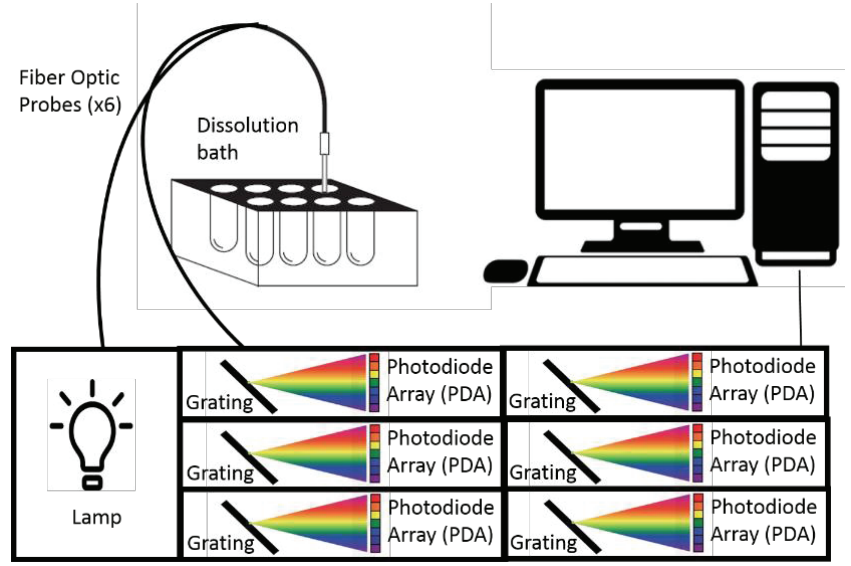

C

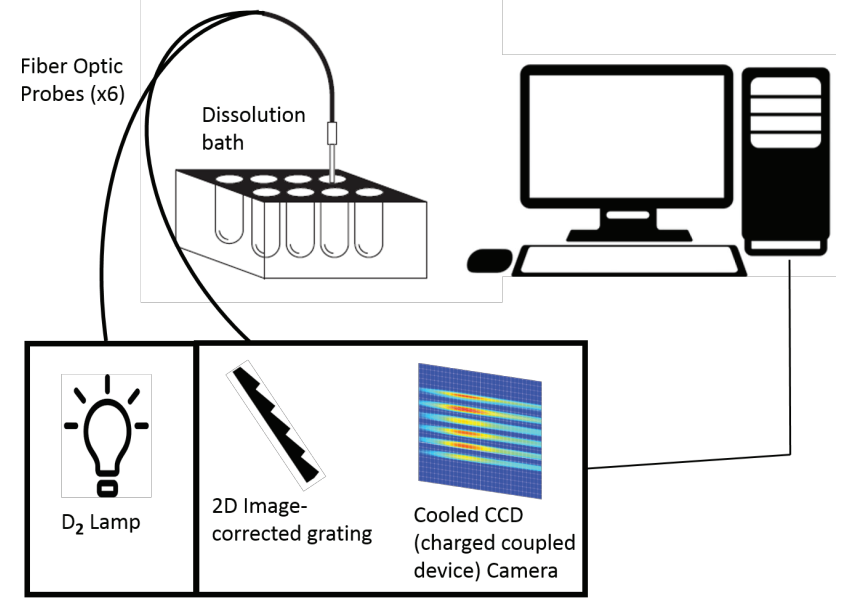

Figure 1. Different configurations of in situ UV systems. (A) Multiplexor and scanning monochromator. (B) Multiple photodiode array spectrographs. (C) Imaging spectrograph and CCD.

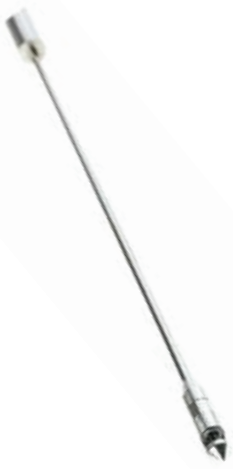

C

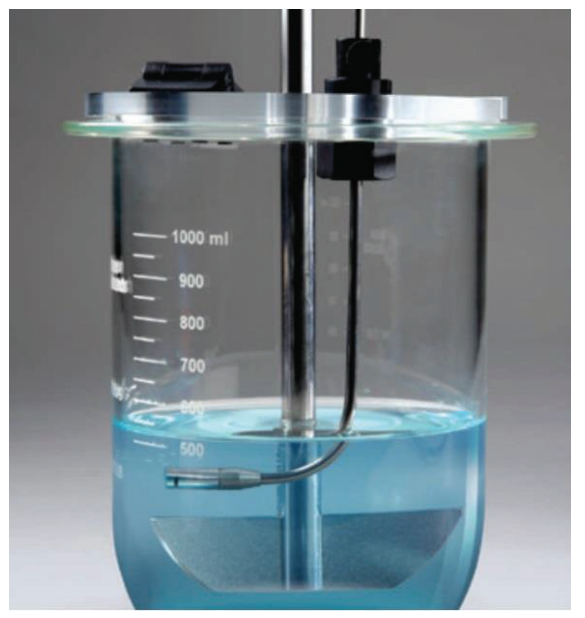

D

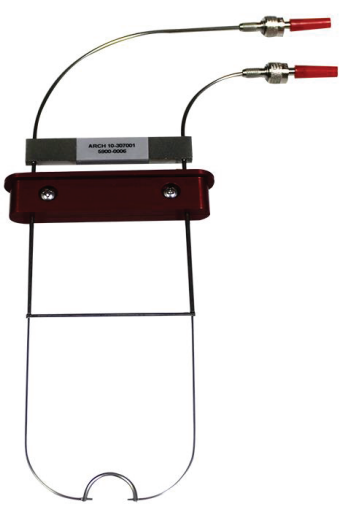

Figure 2. Different probes utilized in in situ UV systems. (A) Conventional transflectance probe. (B) Optimized transflectance probe. (C) J-Probe. (D) Arch probes. 
on two sides of the opening to one. This also reduced the bubble and particulate trapping (18).

Another recent design also bends the probe 90 degrees to create a horizontal sampling volume, which further reduce the chance of trapping bubbles and particles. One drawback of this design is that because the probe is no longer rotationally symmetric, it is not possible to align the opening of the probe the same way in all vessels and for all pathlength tips. This can be an issue since the literature contains examples of dependence of dissolution rates on probe orientation. A very interesting design was also developed that featured two simultaneous pathlengths to expand the range of concentrations that can be measured in one dissolution test (22).

Another probe design was developed specifically for dissolution testing. The probe uses curved input and output legs facing each other and separated by a fixed gap that becomes the pathlength (16). This design yields the thinnest probe with a horizontal gap that is the least likely to capture bubbles or particles. Because the gap is equal to the pathlength in this design, and because of the small size that the liquid has to transverse through, this design supports pathlengths as short as $0.25 \mathrm{~mm}$, allowing measurement of solutions four times as concentrated as other probes. Drawbacks of this design include limits on the maximum pathlength to $10 \mathrm{~mm}$ (versus $20 \mathrm{~mm}$ for dip probes) because there are no focusing optics, and the fact that the pathlength is fixed, meaning a different probe must be used for each pathlength. Also, as is the case with in-vessel temperature probes, the thin portion of the probe inserted into the dissolution medium requires more careful handling.

\section{CHALLENGES OF UNFILTERED MEASUREMENT}

One of the biggest differences between other UV and liquid chromatography (LC) measurements and fiber optic UV measurements is the need to address the challenges of measuring directly in the vessel, where other components such as excipients, undissolved capsules, microspheres, etc. are present and can interfere with the measurement by either absorbing or scattering light. In standard measurements, these challenges are eliminated by first filtering the sample before analyzing it; however, this is not an option for in situ fiber optic systems. Although it would be possible to place a filter over the opening of the fiber optic probe, any blockage of the filter will result in a stagnant volume inside the probe that will not accurately represent the concentration of API dissolved in the rest of the vessel at a given time. This is because unlike manual or automatic liquid sampling with cannulas, there is no suction applied to continuously supply fresh media past the filter.

Instead, one has to use so-called "mathematical filters" to remove the contribution of non-API components. These filters normally take one of two forms (Fig. 3). The first is baseline correction. The concept here is that the contribution of other components to the absorbance measured at the analytical wavelength is modelled by measuring their contribution at a wavelength(s) where the API exhibits no absorbance. These corrections can range from a simple one-point baseline offset correction to more complicated multipoint schemes, including ones that use an exponential baseline to model the wavelength dependences of light scattering from particles.

A

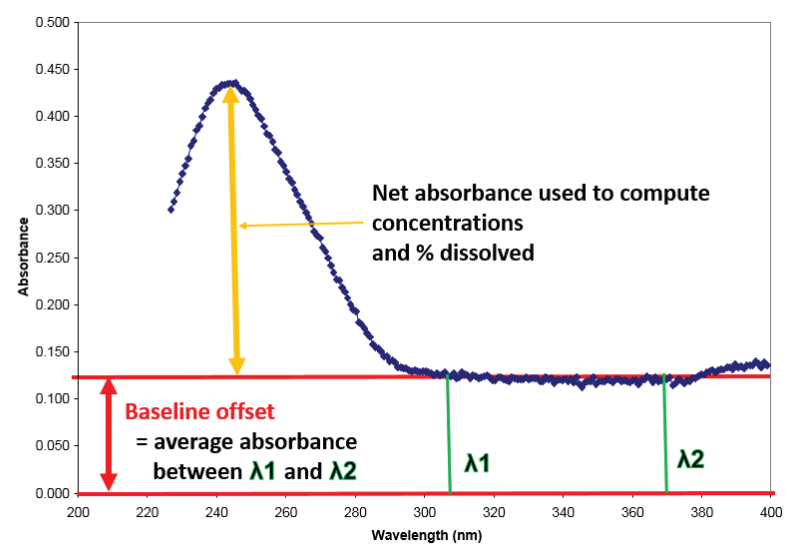

B

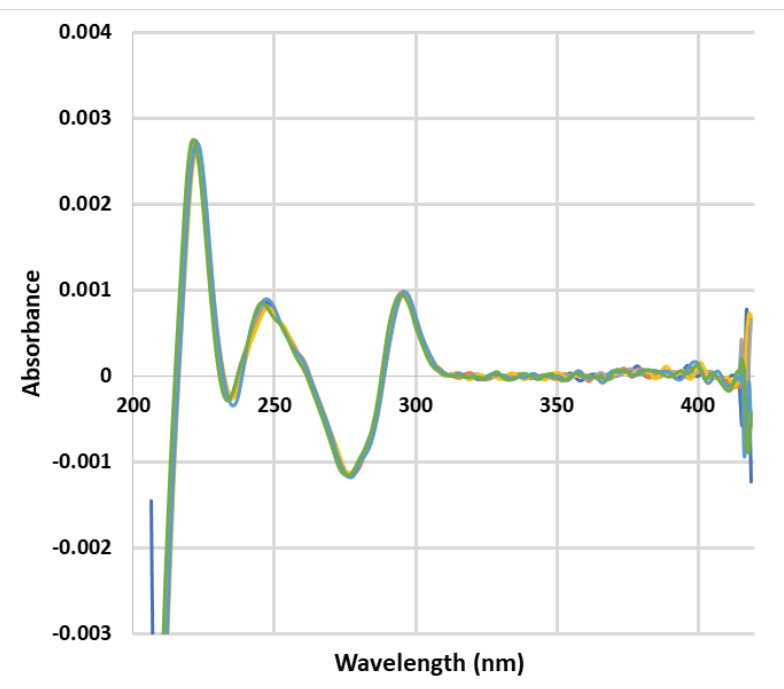

Figure 3. Different "mathematical filters" utilized in in situ UV systems. (A) Baseline correction. (B) Second derivative. 
The second category of correction algorithms is based on derivative spectroscopy. The idea here is essentially that the slope of the API absorbance peak is different from the slope of the interference, and one can exploit this difference to discriminate the contribution of the API from scattering or absorbance from other components. The percent dissolved is computed by comparing the computational derivative value of the absorbance spectra at the analytical wavelength of the sample versus the standard.

A first derivative is enough to eliminate the contribution from a constant baseline shift but suffers from the unfortunate fact of having an amplitude of zero at the analytical peak, so the second derivative is typically used instead. The second derivative goes back to having a peak at the analytical wavelength and can also discriminate between the API spectrum and a complex contribution, such as exponential light scattering from another component. Derivative filtering is particularly useful when there is no convenient wavelength where the API does not absorb but the other components still contribute, or in cases where the contribution from other components is spectrally complex. The drawback to derivative filtering is that since the derivative spectra are produced computationally rather than in closed form, they have reduced the signal-to-noise ratio. This reduction is even greater for the second derivative. A consequence of this reduced signal-to-noise ratio is that the measurement is less sensitive, i.e., the minimum concentration and label claim that can be correctly quantified is increased. This limitation can be somewhat overcome by applying spectral smoothing. This comes at the expense of effective resolution, but given the broad width of pharmaceutical API peaks, this is normally not an issue.

\section{MULTICOMPONENT ANALYSIS}

Traditionally, analysis of multicomponent (multiple API, multiple interfering components, matrix effects, etc.) samples in dissolution have been relegated to LC analysis. However, work predating LC and subsequent methods have been developed to apply UV to these samples. In situ UV testing is particularly suitable for these methods because what they typically require is complete spectra at each time point collected as close to simultaneously as possible. Many chemometric methods such as PLS and PCR have been applied to this problem. Most tend to be limited in utility, especially when the multiple components exhibit a "matrix effect", where the total spectrum is not a simple superimposition of the API spectrum and the other components spectra. Instead, the constituents' spectra themselves are altered by the concentration of the other components.

Again, two computational methods are employed to extract the dissolution profiles of multiple components in in situ UV. The first is the derivative zero crossing method (23). The idea of this method is that the derivatives of the spectra of the different components have azero value (zero crossing) at different wavelengths, so, at that wavelength, they do not contribute to the derivative spectrum of the mixture. Using a two-component mixture as an example, one measures the concentration of component " $\mathrm{A}$ " by analyzing the value of the mixture at a wavelength where component " $\mathrm{B}$ " has a zero crossing, and vice versa. The drawback of this technique is that if the two components have similar spectra, the signal of the other component at the zero crossing of the first tends to be low, making accurate quantitation difficult. This is further acerbated by the fact that data from only one or a few wavelengths are used in the calculation (Fig. 4).

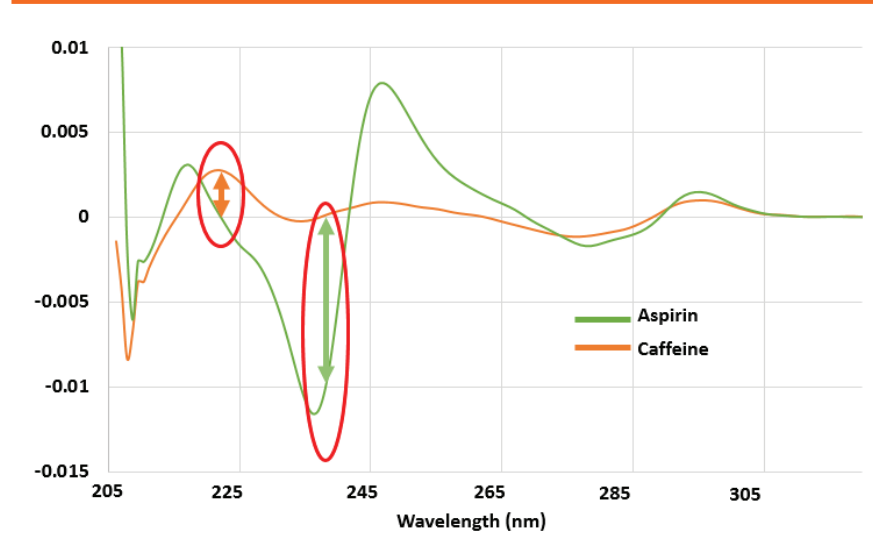

Figure 4. Zero crossing derivative spectra for mathematically resolving multicomponent dissolutions.

The second method, called classical least squares (CLS), addresses this issue. CLS is broadly used to solve problems where some function " $C$ " depends on " $A$ " and " $B$ ", but " $A$ " and " $B$ " also depend on each other $(24,25)$. The way CLS works is that one first measures some known mixtures of " $\mathrm{A}$ " and " $\mathrm{B}$ ", which become a training set for the algorithm. The algorithm is closed form, so always yields a unique answer to a given set of measurements. It is simply based on using matrix math to solve the problem of which mix of " $A$ " and " $B$ " would most closely yield the observed combined spectrum at all wavelengths analyzed. Because it considers as many spectral points as possible, this algorithm works for a wider range of pharmaceutical products. The only added complexity in 
its use is the fact that one must typically prepare two or three extra standards (Fig. 5).

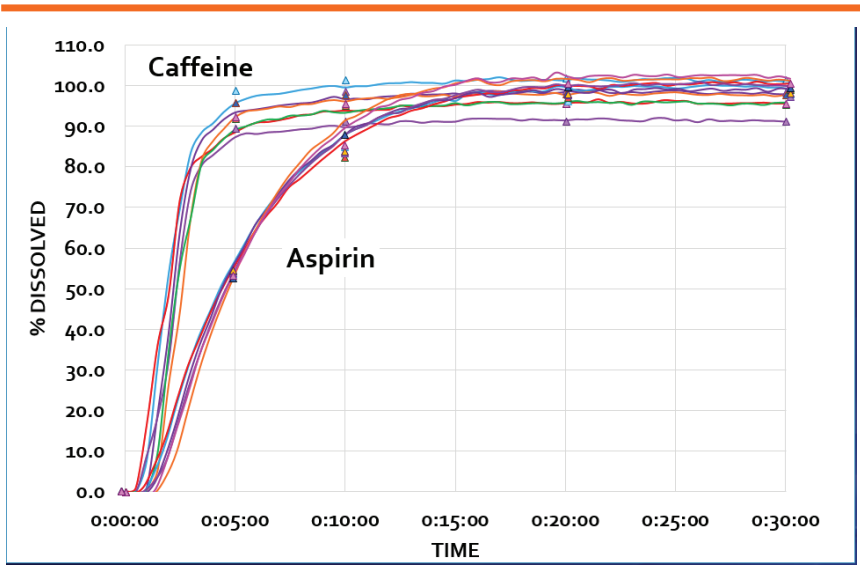

Figure 5. Comparison of multiple APIs mathematically resolved using CLS vs. HPLC.

API, active pharmaceutical ingredient; CLS, Classical Least Squares; HPLC, high-performance liquid chromatography.

\section{PRESENT SITUATION OF QC DISSOLUTION}

As with any new technique, the industry has shown caution in the acceptance of UV fiber optic dissolution for QC and drug product batch release. From a regulatory perspective, in situ UV dissolution analysis has always been regarded as simply an alternative way of doing conventional UV (26). USP Chapter <1092> The Dissolution Procedure: Development and Validation Spectrophotometric Analysis Section explicitly states that "fiber optics as a sampling and determinative method, with proper validation, is an option." The FDA has also recognized the technique in its own internal training as early as 2004. Finally, both the FDA and USP have directly purchased and been using in situ UV dissolution systems internally for nearly two decades.

Currently, there are many hundreds of commercial in situ UV systems in use in the pharmaceutical industry. These include units utilized in research and development, formulation, stability, analytical, and QC groups. The use of fiber optic-based in situ UV dissolution testing has been validated in multiple studies comparing results with established techniques such as conventional UV and LC. Furthermore, suitability has been documented by the implementation of hundreds of systems worldwide, including at regulatory agencies such as the FDA and USP. There are multiple products around the world being released based on dissolution testing performed using in situ UV systems. For some of these products, the in situ method was the primary method documented in the original NDA filing. These include ximelagatran (Exanta) from AstraZeneca and venlafaxine (Effexor) from Pfizer (of course, the description in the monographs simply reads
UV, and an analytical wavelength and the UV method like diode array versus scanning, is never specified). Others have been released with in situ UV as a secondary method, such as oxycodone (Oxycontin) from Purdue Pharma. Another application involved the use of in situ UV for a two-step tier-2 dissolution of over-encapsulated erlotinib (Tarceva) tablets (27).

\section{APPLICATIONS IN BIO-PREDICTIVE DISSOLUTION}

In situ UV dissolution has found its niche in bio-predictive dissolution, which serves the need for API characterization and drug product formulation development. Alternative techniques are more readily adopted in this area due to its non-GMP status and not being subject to regulatory compliance requirements. The focus is to evaluate the physicochemical properties of the API, assess biopharmaceutics risks, screen formulation prototypes, perform relative comparison and trending analysis, and generate data for decision making in a timely fashion. UV fiber optic systems have become an ideal choice for meeting these needs.

An early application commercialized by Delphian Technologies was monitoring concentrations of API in small volumes $(2-20 \mathrm{~mL})$. Their solution, an in situ fiber optic UV monitoring system with real time data display, was suitable for evaluation of various effects on solubility, intrinsic dissolution rate, dissolution profiles, supersaturation, precipitation profiles, etc. (28). The UV fiber optic probes were flexible and durable stainless-steel dip probes with variable pathlengths. Due to the small volume and floating particulates, possible particulate accumulation on the surface of the probe that may affect UV detection needs to be considered during experiments. A further innovation on the small volume apparatus was to combine the dissolution experiment with transmembrane permeation (Fig. 6), which enabled evaluation of the formulation dissolution performance and the drug absorption potential and flux at the same time (9). For such an apparatus, a donor chamber and a receiver chamber are connected through a membrane. Two fiber optic probes are mounted in these two chambers (one in each) to measure the drug concentration in the chambers over the time.

UV fiber optics systems have also been applied to biphasic dissolution, an alternative testing methodology that integrates the in vitro dissolution of a drug in an aqueous phase and its subsequent partitioning into an organic phase to simulate the in vivo drug absorption. A biphasic dissolution system consists of two immiscible phases: an 
aqueous phase and an upper organic phase. In an early application, a UV fiber optic probe was placed in the organic phase, while the detection of drug release in the aqueous phase was performed by withdrawing aliquots for offline measurement (10). A recent application was demonstrated where two sets of fiber optic probes were used, each controlled by a separate UV spectrometer (29). The set of the probes placed in the aqueous phases of the multiple vessels were Arch probes, and the other set of probes placed in the organic phases of the vessels were dip probes (Fig. 7). The in situ UV measurements provide detailed drug release profiles for both phases, helping to improve the understanding of the formulation's performance and make better decisions regarding these

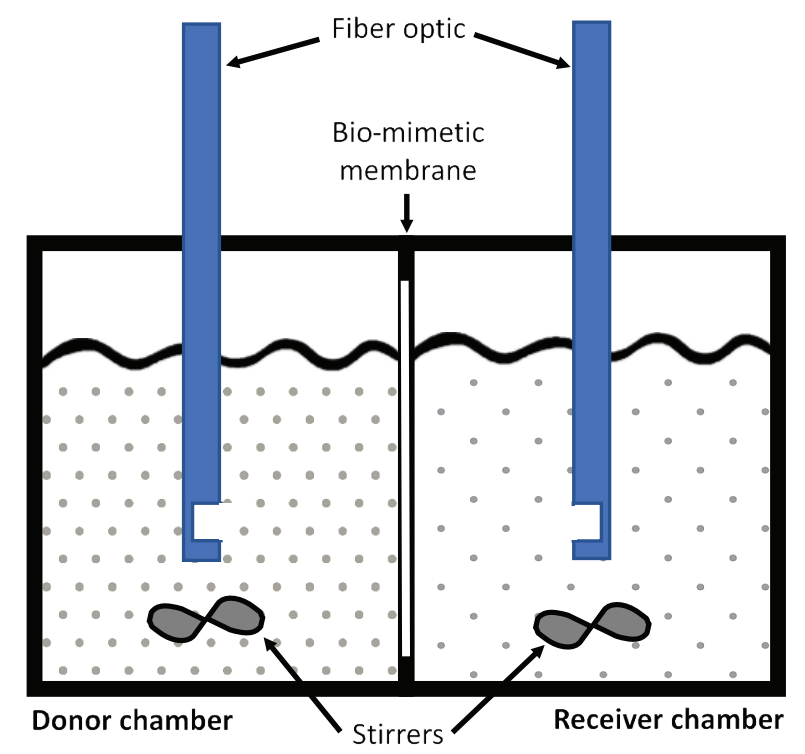

Figure 6. Schematic drawing of pION, Inc. $\mu$ Flux apparatus, with two UV fiber optic probes added to each the donor and receiver chambers.

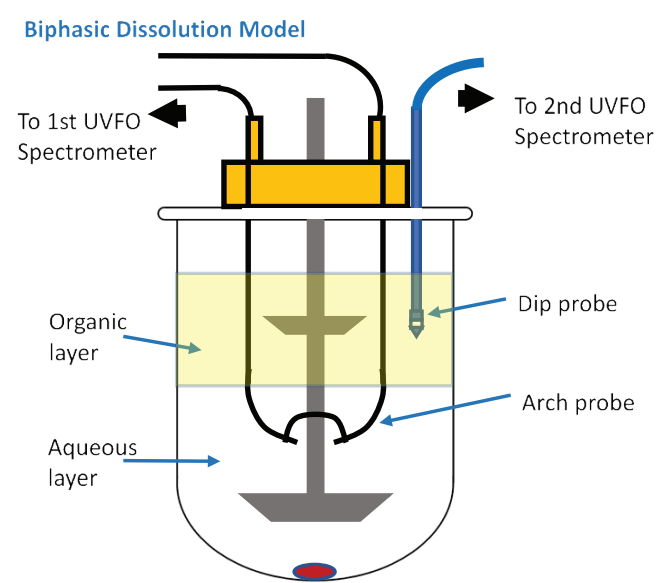

Figure 7. Schematic drawing of biphasic dissolution in USP apparatus 2, with use of two UV fiber optic probes (one Arch probe, and one dip probe) controlled by two UV spectrometers for detection of the drug concentrations in the two phases (29). UVFO, UV fiber optic.

\section{CONCLUSION}

Early adopters of in situ UV fiber optic dissolution testing were drawn to these systems because of the unique capabilities - the ability to produce complete profiles with hundreds of time points, the ability to characterize extremely fast dissolution such as orally dissolving products with several second temporal resolution, or the ability to measure data unattended for multiday or even weeks-long tests. Next came users who needed a solution for samples that could not be addressed by conventional dissolution methods. These included samples that could not be readily filtered, such as nanosuspensions or coated microspheres, where the only option was real-time measurement because it is almost impossible to avoid erroneous results due to continuing release between sample collection and analysis more recently, samples with two APIs or a matrix effect that would otherwise only be possible to analyze by LC.

These non-conventional samples that defy normal dissolution testing will continue to attract first-time users. Far more exciting, however, is the adoption of in situ UV for conventional dosage forms that could be done using other methods. Instead, these dosage forms are validated and manufactured using in situ UV systems because these systems offer the cost and time savings associated with UV analysis, amplified by the lack of need for sampling consumables, such as filters, syringes, lines, and cleaning solvents. In addition, in situ UV systems finally realize the promise of automation where user errors are eliminated and labor is actually reduced by true unattended operation.

\section{ACKNOWLEDGEMENTS}

The authors would like to thank Guy Inman, Jonas Johansson of AstraZeneca, and Vivian Gray of V. A. Gray Consulting, Inc. for insightful discussions and contributions to this paper.

\section{CONFLICT OF INTEREST}

The authors disclosed the following conflict of interest: I. Nir is employed by Distek, Inc., a manufacturer of a commercial fiber optic dissolution system.

\section{REFERENCES}

1. Josefson, M.; Johansson, E.; Torstensson, A. Optical fiber spectrometry in turbid solutions by multivariate calibration applied to tablet dissolution testing, Anal. Chem. 1988, 60, 2666-2671. DOI: 10.1021/ac00175a004.

2. Brown, C. W.; Lin, J. Interfacing a fiber-optic probe to a diode array UV-visible spectrophotometer for drug dissolution tests. Applied Spectroscopy. 1993, 47, 614-618. DOI: 


\subsection{6/0003702934067261.}

3. Chen, C.; Brown, C. A drug dissolution monitor employing multiple fiber optic probes and a UV/visible diode array spectrophotometer. Pharm. Res. 1994, 11, 979-983. DOI: 10.1023/A:1018975002025.

4. Inman, G.; Wethington E.; Baughman, K.; Horton, M. The transition to fiber optic in situ dissolution testing: hydrodynamic effects and sampling techniques. Presented at the AAPS Annual Meeting, Indianapolis, IN, November, 2000.

5. Inman, G. Quantitative assessment of probe and spectrometer performance for a multi-channel CCD-based fiber optic dissolution testing system. Dissolution Tech. 2003, 10, 26-32. DOI: 10.14227/DT100403P26.

6. Schatz, C.; Ulmschneider, M.; Altermatt, R.; Marrer, S.; Altorfer, $\mathrm{H}$. Thoughts on fiber-optic dissolution testing. Dissolution Tech. 2001, 8, 6-11. DOI: 10.14227/DT080201P6.

7. Nir, I.; Johnson, B.; Johansson, J; Schatz, C. Application of fiberoptic dissolution testing for actual products. Pharm. Tech. 2001, 25, 33-41.

8. Zolnik, B. S.; Raton, J.-L.; Burgess, D. J; Application of USP apparatus 4 and in situ fiber optic analysis to microsphere release testing. Dissolution Tech. 2005, 12, 11-14. DOI: 10.14227/ DT120205P11.

9. Borbás, E.; Sinkó, B.; Tsinman, O.; Tsinman, K.; Kiserdei, E.; Démuth, B.; Balogh, A.; Bodák, B.; Domokos, A.; Dargó, G.; Balogh, G. T.; Nagy, Z. K. Investigation and mathematical description of the real driving force of passive transport of drug molecules from supersaturated solutions. Mol. Pharmaceutics 2016, 13, 3816-3826. DOI: 10.1021/acs.molpharmaceut.6b00613.

10. Zhu, A. Z. X.; Ho, D.; Gemski, C. K.; Chuang B.; Liao, M.; Xia, C. Utilizing in vitro dissolution-permeation chamber for the quantitative prediction of $\mathrm{pH}$-dependent drug-drug interactions with acid-reducing agents: a comparison with physiologically based pharmacokinetic modeling. AAPS Journal. 2016, 18, 1512-1523. DOI: DOI: 10.1208/s12248-016-9972-4.

11. Xu, H.; Shi Y.; Vela, S.; Marroum, P.; Gao, P. Developing quantitative in vitro-in vivo correlation for fenofibrate immediate-release formulations with the biphasic dissolutionpartition test method. J. Pharm. Sci. 2018, 107, 476-487. DOI: 10.1016/j.xphs.2017.06.018.

12. Cho, J. H.; Gemperline, P. J.; Salt, A.; Walker, D. S. UV/Visible spectral dissolution monitoring by in situ fiber optic probes. Anal. Chem. 1995, 67, 2858-2863. DOI: 10.1021/ac00113a020.

13. Cho, J. H.; Gemperline, P. J.; Walker, D. S. Wavelength calibration method for a CCD detector and multi-channel fiber optic probes. Appl. Spect. 1995, 49, 1841-1845. DOI: 10.1366/0003702953966055.

14. Bynum, K.; Roinestad, K.; Kassis, A.; Pocreva, J.; Gehrlein, L.; Cheng, F.; Palermo, P. Analytical performance of fiber optic probe dissolution system. Dissolution Tech. 2001, 8, 1-8. DOI:

\subsection{7/DT080401P13.}

15. Bynum, K. New advances in fiber-optic dissolution testing. Presented at Barnett International Conference on Dissolution Testing, Tysons Corner, VA, December 2000.

16. Inman, G.; Wethington, E.; Baughman, K.; Horton, M. System optimization for in situ fiber optic dissolution testing. Pharm. Technol. 2001, 25, 92-100.

17. Earnhardt, J.; Nir I. Fiberoptic dissolution testing advances drug quality control. Spectroscopy 2000, 15, 31-38.

18. Lu, X.; Lozano, R.; Shah, P. In situ dissolution testing using different UV fiber optic probes and instruments. Dissolution Tech. 2003, 10, 6-15. DOI: /10.14227/DT100403P6.

19. Cox, D. C.; Furman, W. B.; Moore, T. W.; Wells, C. E. Guidelines for dissolution testing: an addendum. Pharm. Technol. 1984, 8, 42-46.

20. Wells, C. E. Effect of sampling probe size on dissolution of tableted drug samples. J. Pharm. Sci. 1981, 70, 232-233. DOI: 10.1002/jps.2600700234.

21. Savage, T. S.; Wells, C. S. Automated sampling of in vitro dissolution medium: effect of sampling probes on dissolution rate of prednisone tablets. J. Pharm. Sci. 1982, 71, 670-673. DOI: 10.1002/jps.2600710616.

22. Johansson, J.; Cauchi, M.; Sundgren, M. Multiple fiber-optic dual-beam UV/Vis system with application to dissolution testing. J. Pharm. Biomed. Anal. 2002, 29, 469-476. DOI: 10.1016/ S0731-7085(02)00091-2.

23. Patel, K. N.; Patel, J. K.; Rajput, G. C.; Rajgor, N. B. Derivative spectrometry method for chemical analysis: a review. Der Pharmacia Lettre. 2010, 2, 139-150.

24. Draper, N. R; Smith, H. Applied Regression Analysis, 3rd ed.; John Wiley \& Sons: New York, 1998, p 21. DOI:10.1002/9781118625590.

25. Sharaf, M. A.; Illman, D. L.; Kowalski, B. R. Chemometrics, $1^{\text {st }}$ ed.: John Wiley \& Sons: New York, 1986, pp 135-137. DOI: 10.1002/ jcc.540090712.

26. Gray, V. Dissolution testing using fiber optics - a regulatory perspective. Dissolution Tech. 2003, 10, 33-36. DOI: 10.14227/ DT100403P33.

27. Lu, X.; Xiao, B.; Lo, L.; Bolgar, M. S.; Lloyd, D. K. Development of a two-step tier-2 dissolution method for blinded overencapsulated erlotinib tablets using UV fiber optic detection. J. Pharm. Biomed. Anal. 2011, 56, 23-29. DOI: 10.1016/j.jpba.2011.04.026.

28. Avdeef, D.; Voloboy, A; Foreman, Dissolution-solubility: pH, buffer, salt, dual-solid, and aggregation effects. In Comprehensive Medicinal Chemistry II, ADME-TOX Approaches; Vol. 5; Testa, B.; van de Waterbeemd, H.; Eds.; Elsevier: Oxford, UK, 2007, pp 415-416.

29. Lu, X.; Lo, L.; Grove, G.; Renslow, B. Evaluation of use of dual paddles for biphasic dissolution systems for poorly-soluble drugs, Presented at the AAPS Annual Meeting, Denver, CO, Nov 13-17, 2016. 